\title{
Stress Tests and VaR Analysis in the Process of Risk Management
}

\section{Viviane Y Naimy*}

Faculty of Business Administration and Economics, Notre Dame University, Louaize - Lebanon, Lebanon

The stress tests are important components of the risk management process. What the traditional VaR analysis disregarded in terms of possible extreme scenarios, is in fact well measured by the stress tests. Therefore stress tests did not replace the VaR; they have successfully assessed the sensitivity of businesses to sharp changes in asset prices. Is it reasonable and feasible to integrate the stress tests to the VaR analysis?

The most important lesson given by the financial crisis of 2007 is the significance of the stress tests together with the mechanics of the VaR models. Such models are important however based on historical approach while risk management deals with the future. Consequently, the managerial assessment is necessary to develop credible extreme scenarios that can eventually serve as a base for the stress tests. After gauging all these possible scenarios, the financial institution can then undertake the appropriate preventive measures to reduce the impact of the most unfavorable scenarios. An unquestionable advantage of this approach is a better understanding of the very nature of risks [1].

\section{Generating Scenarios}

The most often used approach for the VaR calculation is the historical simulation. According to this approach, historical data represent a good indicator of future achievements (over one to ten days). If a certain event is not included in the data history, it won't affect the VaR results when the latter is calculated according to the traditional bases. Taking the volatility into consideration, it leads to excessive results when the market is volatile. The extreme values theory allows smoothing and spreading the losses of the distribution's tails. The VaR calculation based on extreme scenarios constitutes a promising development; however, the historical nature of the calculation will always remain a handicap against such trial. Possible events non- covered by historical data are not considered. The stress tests are there to solve this problem and overcome this weakness of the VaR measurement.

The stress test means the expectation of the portfolio changes during extreme moves in several different asset prices (for example, equity prices, exchange rates, interest rates, and interest rate spreads). This can be divided into two steps:

\section{The development of extreme market variation scenarios.}

2. The evaluation of the portfolio according to these scenarios.

Sometimes these changes are measured in terms of standard deviations. If the daily changes are distributed normally, a daily change of five standard deviation can happen once each 7,000 years. Practically, it is not impossible or rare for such event to happen once or twice each 10 years. This shows that the hypothesis of a normal distribution is not appropriate in the risk management practices.

The key element of the stress tests is the choice of scenarios. Below are some possible procedures:

\section{Stress tests on individual variables:}

This means the use of scenarios modifying one variable while keeping the remaining ones constant. For instance, a parallel shift in the yield curve of a 100 basis points, a $20 \%$ change of the implied volatility of a given asset, a $5 \%$ increase in the exchange rate of a major currency, etc. The impact of small changes in one variable is measured by its delta and the impact of more important variations can be measured by delta and gamma. In the case of sharp moves, important variations inhibit the use of the "Greeks" for risk assessment.

\section{Scenarios based on many variables}

When a particular asset price witnesses a large change or a shock, other tightly linked variables will also react to this change. This leads the institutions to develop scenarios according to which many variables move at the same moment. Such scenarios are then based on significant market moves in the past or on plausible market event that has to happen.

\section{Scenarios conducted by managers}

History cannot be repeated typically the same way because people are conscious of the past financial crisis and try to avoid committing the same mistakes. It is not possible for the 2007 subprime financial crisis to be repeated again however, that does not mean that other credit crisis cannot take place in the future. The most efficient scenarios are those generated by managers. In fact, they can easily use their knowledge of the markets and the economic situation surrounding them in order to draw the scenarios that might lead to serious losses. Sometimes these scenarios are based on past events, however completed with key elements linked to the current financial and economic conditions that take into consideration the systemic risk.

\section{Inverted stress tests:}

Inverted stress tests mean the design of scenarios generating important losses. If for instance ten variables are identified, the changes of other variables can be conditionally specified with reference to the changes in the key variables. Standard algorithms can be used to find in a space of two dimensions the combinations of the ten variables' variations that generate the worst results. The disadvantage of the inverted test is that it can produce unrealistic scenarios. Conversely, this procedure allows screening some scenarios difficult to be assumed or thought of by managers.

\section{Integrating the Stress Tests with the VaR}

According to Berkowitz [2] the stress tests would have been more serious if integrated to the VaR calculation by assigning a probability to the tests scenarios. Unfortunately the estimation of a subjective probability of a rare event is delicate for all experts. In order to

${ }^{*}$ Corresponding author: Viviane $\mathrm{Y}$ Naimy, Faculty of Business Administration and Economics, Notre Dame University, Louaize - Lebanon, Lebanon, E-mail: vnaimy@ndu.edu.lb

Received July 26, 2012; Accepted July 28, 2012; Published July 31, 2012

Citation: Naimy VY (2012)Stress Tests and VaR Analysis in the Process of Risk Management. J Bus \& Fin Aff 1:e114. doi:10.4172/2167-0234.1000e114

Copyright: (C) 2012 Naimy VY. This is an open-access article distributed under the terms of the Creative Commons Attribution License, which permits unrestricted use, distribution, and reproduction in any medium, provided the original author and source are credited. 
Citation: Naimy VY (2012) Stress Tests and VaR Analysis in the Process of Risk Management. J Bus \& Fin Aff 1:e114. doi:10.4172/2167$0234.1000 \mathrm{e} 114$

Page 2 of 2

facilitate this task, the stress committee can classify each scenario in a category with a predefined probability. Only then, the stress tests can be smoothly integrated to the analysis of the VaR. Unfortunately, this suggestion was not considered in the consultative document issued by the Basle Committee [3].

\section{Reference}

1. Kupiec PH (1998) Stress-Testing in a Value at Risk Framework. The Journal of Derivatives 6: 7-24.

2. Berkowitz J (1999) A Coherent Framework for Stress-Testing. FEDS Working Paper 99-29.

3. Basle Committee on Bank Supervision (2009) Principles for Sound Stress Testing. Practices and Supervision-consultative paper. 